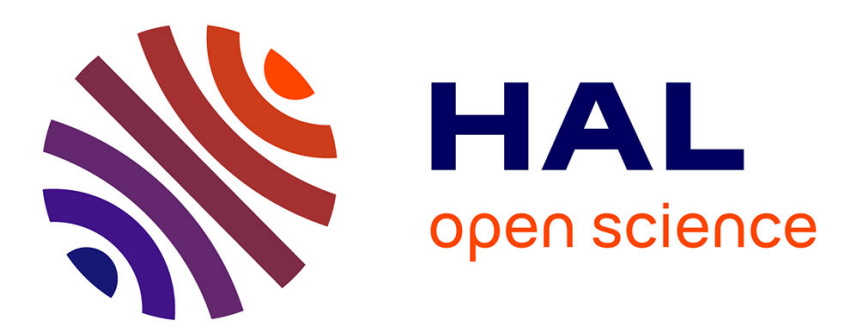

\title{
La délibération comme démarche réflexive accompagnant la décision médicale
}

\author{
Eric Delassus
}

\section{To cite this version:}

Eric Delassus. La délibération comme démarche réflexive accompagnant la décision médicale. Éthique publique - revue internationale d'éthique sociétale et gouvernementale , 2015, Éthique et reconfigurations de l'économie de marché: nouvelles alternatives, nouveaux enjeux, 16 (2), http://ethiquepublique.revues.org/1569. hal-01172054

\section{HAL Id: hal-01172054 \\ https://hal.science/hal-01172054}

Submitted on 6 Jul 2015

HAL is a multi-disciplinary open access archive for the deposit and dissemination of scientific research documents, whether they are published or not. The documents may come from teaching and research institutions in France or abroad, or from public or private research centers.
L'archive ouverte pluridisciplinaire HAL, est destinée au dépôt et à la diffusion de documents scientifiques de niveau recherche, publiés ou non, émanant des établissements d'enseignement et de recherche français ou étrangers, des laboratoires publics ou privés. 


\title{
Éthique publique
}

Revue internationale d'éthique sociétale et gouvernementale

vol. $16, \mathrm{n}^{\circ} 2$ | 2014 :

Éthique et reconfigurations de l'économie de marché : nouvelles alternatives, nouveaux enjeux

Zone libre

\section{La délibération comme démarche réflexive accompagnant la décision médicale}

\author{
ÉRIC DeLASSUS
}

\section{Résumés}

Français English

La délibération est souvent perçue comme l'œuvre d'une liberté examinant de manière autonome les éléments qui conduisent à la prise de décision, elle-même perçue comme le moment premier de l'action. Cette vision des choses n'est-elle pas la conséquence d'une illusion rétrospective ? Le processus décisionnel dans lequel s'inscrit la délibération ne doit-il pas plutôt être envisagé comme un enchaînement causal par lequel les acteurs sont emportés sans être véritablement les auteurs du scénario auquel ils participent ? Une telle approche déterministe doit-elle pour autant nous conduire à renoncer à « reprendre la main » sur nos décisions?

L'analyse qui est ici conduite porte sur la décision dans le domaine médical. Elle s'inspire de la pensée de Spinoza et tend à montrer que même si nous sommes déterminés, la puissance réflexive de l'esprit est en mesure de mieux éclairer et orienter les différents protagonistes de la délibération et de la décision médicales.

Deliberating is often regarded as the work of freedom that independently examines the factors leading to decision-making, which is then perceived as the first step leading to action. Is this interpretation resulting from a retrospective illusion? Should the process of decision-making, 
which deliberation is a part of, rather be considered as a causal chain through which the actors are carried away without being truly the authors of the scenario in which they participate, or not ? Should a deterministic approach lead us so far as to giving up "taking control" of our decisions? Here the analysis focuses on decision-making in the medical field. It is drawn upon Spinoza's thoughts and suggests that however determined we may be, the reflexive power of the mind is able to better inform and guide the various actors of deliberation and medicalmaking.

\section{Entrées d'index}

Mots-clés : délibération, décision médicale, déterminisme, Spinoza

Keywords : deliberation, medical decision, determinism, Spinoza

\section{Texte intégral}

\section{La rupture et la continuité}

La décision est souvent perçue comme étant à la fois un aboutissement et un commencement, aboutissement d'une délibération qui la précède et commencement d'une action qui résulte d'un choix réfléchi. On peut cependant s'interroger sur le bienfondé d'une telle perception des choses. En effet, si toute action est le fruit d'une décision - et ce que l'on dit de l'action vaut aussi pour l'inaction, ne pas agir, c'est décider de ne rien faire -, toute décision n'est pas nécessairement le fruit d'une délibération. Ainsi, la décision médicale qui doit fréquemment être prise dans l'urgence ne résulte pas toujours d'une réelle délibération. Le médecin ou l'équipe soignante doivent agir au plus vite et se référer à des modes opératoires faisant intervenir l'habitude, l'expérience, l'intuition, termes qui sont souvent perçus comme opposés à une rationalité s'exerçant par l'analyse et la réflexion dont on a coutume de croire qu'elles sont au cœur même de toute véritable délibération.

Le plus souvent, la décision résulte d'un enchaînement d'affects et elle n'est finalement qu'un maillon de la chaîne qui constitue l'action, plutôt qu'une rupture qui l'engage. Le terme de décision renvoie à l'idée de trancher, de couper, de scinder, on y trouve la même racine étymologique que dans l'incision qui consiste à entailler un corps, mais sans le diviser totalement, pour accéder à son intériorité :

\section{Décider au sens originaire du terme, c'est « couper en tranchant ». Aujourd'hui encore, le vocable « décision » évoque assez spontanément, dans nos esprits, une initiative saillante qui trouve sa forme prototypique dans le geste du chirurgien qui se résout à trancher dans la chair du patient avec son bistouri. Celui qu'on nomme le « décideur » est capable, quand il le faut, de donner le coup de sécateur qui tranchera dans un débat en faveur d'une des branches de l'alternative (Le Coz, 2007 : 23).}

À la différence de l'incision, la décision suppose, quant à elle, une rupture, une séparation, non plus spatiale, mais temporelle : il y aurait l'avant et l'après de la décision, l'avant de la délibération et l'après de l'action. Nous pouvons cependant nous demander, à la lumière de ce que nous enseigne la décision médicale prise dans l'urgence, si ce schéma n'est pas le fruit d'une illusion rétrospective. Ne percevons-nous pas finalement la décision comme la flèche de Zénon « qui vibre, vole, et qui ne vole pas ! » en figeant par l'imagination ce qui dans la réalité s'inscrit dans un mouvement 
indivisible ? En conséquence, décision et délibération ne seraient pas si distinctes qu'il y paraît l'une de l'autre et la rationalité qui présiderait à leur effectuation ne serait pas non plus sans rapport avec les affects - impressions, sentiments, émotions - que l'on ne peut facilement évacuer, principalement lorsque l'on est face à l'un de ses semblables qui souffre et dont la vie est en jeu.

Toute décision, qu'elle soit prise dans l'urgence ou non, ne s'inscrit-elle pas finalement dans une telle continuité ? N'est-elle pas comparable à l'événement historique qui apparaît comme ce à partir de quoi tout s'engage, mais qui n'est finalement que le jaillissement superficiel issu de mouvements plus profonds s'inscrivant dans une plus longue durée ? La délibération ne serait alors que la manifestation de ces mouvements dont nous n'aurions finalement qu'une conscience très partielle. Autrement dit, la décision ne se réduirait pas à ce qui est décidé, mais au processus par lequel on décide.

Mais, si la liberté qui se trouve à l'œuvre dans la délibération présente un caractère illusoire, elle n'est pas non plus totalement évacuée par notre analyse. Toute pensée qui prend conscience de ses limites est toujours, par cette prise de conscience, en mesure de les dépasser. Il semble donc nécessaire de procéder ici à une analyse des mécanismes qui vont de la délibération à la décision et de la décision à l'action pour accroître cette conscience et peut-être se donner les moyens de mieux décider.

\section{La délibération et la nécessité}

Penser la délibération et la décision qui en découle comme un enchaînement causal continu pourrait nous conduire à envisager, à l'instar de Thomas Hobbes dans son Léviathan, la délibération comme un simple jeu de forces entre des affects, la décision exprimant la victoire de l'affect le plus puissant :

\footnotetext{
Quand, dans notre esprit, appétits et aversions, espoirs et craintes au sujet

d'une seule et même chose arrivent alternativement : quand plusieurs conséquences bonnes et mauvaises nous viennent à l'esprit au sujet d'une chose qu'on se propose de faire ou qu'on omet de faire, de sorte que tantôt nous éprouvons de l'appétit, tantôt de l'aversion : tantôt nous espérons être capables de la faire, tantôt nous désespérons ou craignons de nous engager à la faire toute la somme de désirs, aversions, espoirs et craintes éprouvés jusqu'à ce que la chose soit faite ou qu'on pense impossible à faire s'appelle DÉLIBÉRATION (Hobbes, 2000 : 137-138).
}

Hobbes s'oppose ici de toute évidence à la conception aristotélicienne qui ne conçoit la délibération qu'au sujet des affaires contingentes qui nous concernent. Selon Aristote, on ne délibère pas « sur les choses qui sont en mouvement mais se produisent de la même façon, soit par nécessité, soit par nature, soit par quelque autre cause », pas plus que sur la « meilleure forme de gouvernement pour les Scythes » (1990:133), si on est Lacédémonien.

C'est pourquoi, en bon matérialiste qu'il est, Hobbes oppose à cette conception de la délibération un déterminisme des affects qui évacue de " ce que nous appelons délibération » toute possibilité d'y voir l'exercice d'une quelconque forme de liberté.

Ce que le philosophe anglais ne semble pas voir ici et que verra plus clairement Spinoza, c'est que la prise de conscience d'un tel mécanisme est également productrice d'affects qui nous permettent d'y voir plus clair dans nos processus de décision et de progresser vers la liberté. En effet, tous les affects ne se valent pas. Il est des affects qui nous sont utiles et nous orientent vers ce qui accroît notre puissance, il en est d'autres 
qui la diminuent. Si les premiers sont toujours le jeu des causes externes qui sont fluctuantes et nous font réagir plutôt qu'agir, les seconds, bien qu'ils puissent également procéder des aléas de la fortune, ne peuvent que nous indiquer la meilleure voie à suivre lorsqu'ils sont issus de la raison. La question est alors de savoir comment passer de la soumission à une nécessité externe à la libre manifestation de la nécessité interne.

\section{Le pouvoir de la réflexion}

Si l'on définit la raison comme l'expression de la causalité universelle sous l'attribut de la pensée, ce passage ne peut se faire que par la compréhension des automatismes affectifs et intellectuels qui sont à l'œuvre dans la prise de décision. Être libre, accéder à l'autonomie morale et intellectuelle, atteindre la perfection consiste selon Spinoza à connaître "l'union que l'esprit a avec la nature tout entière » (1999 : 29). Il semble donc indispensable ici de mettre en action notre capacité de penser ${ }^{1}$, qui n'est pas simplement capacité de produire des représentations. Penser, ce n'est pas seulement avoir des idées, c'est également avoir des idées de ces idées. Autrement dit, nous pouvons par la réflexion comprendre pourquoi nous pensons et ressentons ce que nous pensons et ressentons, et lorsque nous l'avons compris réellement, nous ne pensons plus ni ne ressentons les mêmes choses.

Il est donc permis d'envisager la délibération comme l'acte de la pensée par lequel nous nous efforçons de comprendre comment ont pu être prises certaines de nos décisions afin de les rediriger au besoin.

\section{Regarder en arrière pour mieux décider}

Il est certes impossible de délibérer sur le passé, au sens où il n'est pas en notre pouvoir de faire que n'ait pas eu lieu ce qui s'est effectivement produit : je ne peux décider par exemple que César n’a pas franchi le Rubicon. En revanche, il est possible de délibérer à partir du passé, si l'on entend par là réfléchir sur les conditions qui ont donné lieu à la décision. Ainsi, dans le cadre de la délibération et de la décision médicales, il peut sembler particulièrement utile de réexaminer, lorsque l'urgence ne se fait plus sentir, les mécanismes qui ont pu conduire à une action pour réorienter les choix qui ont été faits ou éclairer de futures décisions qui devront être prises dans des conditions similaires. Il s'agit donc, en quelque sorte, de penser la délibération comme la saisie du mouvement immanent qui préside à la décision. Délibérer relèverait donc d'une certaine capacité du médecin à tirer des leçons de l'expérience, non pas dans un sens empiriste en procédant par induction, mais en procédant au contraire par déduction. Il s'agit d'une expérience reprise par l'intellect qui l'analyse pour y saisir la logique immanente et nécessaire qui l'a produite.

Il n’y aurait donc rien d'illégitime à recourir à ce que l'on pourrait paradoxalement nommer une délibération rétrospective, c'est-à-dire non pas une réflexion qui précède et détermine le choix, mais un examen qui en est la conséquence et qui est analyse des déterminations du choix. Cet examen n'aura certes pas d'incidence directe sur les décisions déjà prises, mais pourra éventuellement en corriger l'orientation et surtout déterminer les futures décisions. C'est donc en ce sens qu'il peut malgré tout s'agir 
d'une délibération, dans la mesure où il s'agit de débattre des éléments qui ont pu conduire à la décision pour la réorienter, au besoin, et apporter un éclairage nouveau sur les décisions ultérieures.

Cependant, une fois posés les principes d'une telle délibération, il faut ensuite déterminer les modalités d'effectuation d'une telle démarche.

\section{Qui délibère ? Qui décide ?}

La délibération dans le domaine du soin et de la médecine ne se fait jamais seul. C'est toujours collectivement que l'on délibère, avec une équipe regroupant le plus souvent médecins, soignants et spécialistes de disciplines paramédicales (kinésithérapeutes, diététiciens, psychologues, etc.) et à l'intérieur de ce que Jacqueline Lagrée qualifie de relation triangulaire entre le médecin, le malade et ses proches (2002:186).

C'est d'ailleurs le rapport entre ces trois termes qu'il nous faudra tenter d'éclaircir pour mieux comprendre ce champ de forces qui se constitue dans le cadre d'une délibération collective.

En effet, l'équipe soignante à laquelle s'ajoutent le malade et ses proches ne forme en rien un tout organique pouvant décider comme une seule personne et formant une collectivité parfaitement solidaire. La délibération qui précède, accompagne ou poursuit la décision est le plus souvent l'occasion de tensions et d'opposition entre des forces que personne ne maîtrise totalement (regards différents selon la fonction ou la position que l'on occupe dans l'équipe soignante, divergences d'opinions...). D'autre part, à l'issue de la délibération, il y a toujours en fin de compte quelqu'un qui décide au nom de tous et qui tire une légitimité de la manière dont il a su s'intégrer au processus de décision. Parfois, il détient l'autorité institutionnelle, mais ce n'est pas toujours le cas : qu'il soit médecin, soignant ou le malade lui-même, il est celui qui est parvenu à se faire reconnaître par tous les participants à la délibération une autorité morale qui lui donne droit d'effectuer la synthèse des différents points de vue exprimés et d'en dégager l'orientation que va impliquer la prise de décision. On peut donc aborder sous cet angle la délibération à la fois dans ses aspects psychosociologiques et aussi en un certain sens "politique » puisque s'y introduisent des enjeux de pouvoir dont il importe de fonder la légitimité. La question se pose donc de savoir non seulement qui décide, mais aussi qui, au bout du compte, incarne la décision. Qui est celui à qui peut être imputée la responsabilité des orientations qui ont été choisies ?

Il semblerait donc judicieux de laisser quelque peu de côté les dimensions épistémologiques et psychologiques de la démarche délibérative et des mécanismes de la décision pour se situer sur le terrain de la philosophie politique, qui peut certainement nous en apprendre beaucoup en matière de processus décisionnels. $\mathrm{Si}$ " gouverner, c'est prévoir », c'est justement parce qu'il faut le plus souvent décider dans le cadre de situations pour lesquelles demeure une certaine ignorance des éléments déterminants qui sont à l'œuvre. Aussi importe-t-il, pour mieux canaliser l'ensemble des éléments qui interviennent dans une délibération, qu'ils soient rationnels ou affectifs, individuels ou collectifs, de mettre en place certaines procédures délibératives et décisionnelles pour faire en sorte que tous les acteurs concernés puissent juger celle-ci comme légitime, même si en tant qu'individu leur choix aurait pu s'orienter différemment.

Ainsi pourrons-nous peut-être répondre aux questions qui résument notre analyse précédente : qui délibère ?, qui décide ?, quand et surtout comment? 


\section{Intégrer la délibération dans une démarche procédurale}

Il s'agit donc de définir une procédure de délibération qui permette une décision collective et qui puisse être perçue comme légitime par chacun des participants. La délibération se déroule en faisant intervenir plusieurs points de vue et la décision qui en découle est assumée par tous, mais sans que pour autant chacun acquiesce à tous les jugements qui l'ont déterminée. Le problème de la décision collective se pose donc dans les termes suivants : bien que tous les acteurs ne partagent pas toujours exactement la même vision des choses, il faut prendre malgré tout une décision en procédant de telle sorte que personne ne puisse en contester la légitimité. Il importe cependant de ne pas faire de la procédure de décision un paravent derrière lequel chacun pourrait se cacher pour ne pas avoir à assumer les conséquences de ses choix et de ceux des autres.

Il pourrait sembler paradoxal de recourir à une démarche procédurale intégrant la délibération et évoquant la responsabilité des acteurs de la décision alors que l'on est tout d'abord parti d'une réflexion sur ce que pourrait être la délibération envisagée comme un enchaînement d'affects remettant en cause les notions de volonté autonome et de libre arbitre perçus comme des illusions de la conscience résultant de notre ignorance des mécanismes délibératifs.

Cependant, penser la délibération comme un enchaînement de causes efficientes ne conduit pas pour autant à renoncer à la réflexion et à la responsabilité. S’il faut réfléchir et délibérer, c'est précisément parce que nous ne connaissons pas nécessairement toutes les déterminations qui sont à l'œuvre lorsque nous délibérons et que d'un point de vue pragmatique, nous sommes conduits à toujours faire comme si nous disposions d'un libre arbitre. Notre entendement n'a rien à voir avec l'intellect de Dieu qui en diffère "de toute l'étendue du ciel $»^{2}$, il ne peut donc connaître toutes les déterminations dont il est l'objet. Il peut certes par la réflexion accroître la connaissance des causes qui le déterminent et par là même se constituer comme raison, mais cela n'est pas toujours possible principalement lorsque l'urgence impose la prise de décision. D'autre part, ce déterminisme ne nous dispense pas d'assumer les conséquences de nos actes. Même si nous n'en sommes pas la cause première, nous sommes pris dans un réseau causal. Toute cause est productrice d'effets et nous devons répondre des effets dont nous sommes causes.

Les acteurs de la décision se trouvent donc toujours dans ce type de situation sur la ligne de crête qui sépare l'opinion de la connaissance rationnelle, l'incertitude sur les effets d'un traitement ne pouvant être annulée par la connaissance scientifique du médecin, le doute sur la manière dont une décision sera jugée et dont ses conséquences seront vécues par le patient et sa famille ne pouvant être contrebalancé par un savoir, quel qu'il soit.

Cependant, s'il y a une chose qu'il nous faut savoir, c'est que l'ignorance ne nous invite que rarement au doute : bien au contraire, elle se dissimule fréquemment derrière des idées fictives qui sont les pensées inadéquates des affections que nous ressentons naturellement : " Il est une chose certaine en effet, et nous en avons démontré la vérité dans notre Éthique : les hommes sont nécessairement soumis aux affects, et sont ainsi constitués qu'ils plaignent les malheureux [...] » (Spinoza, 2005 : 93).

Il faut donc tenir compte de ce fait dans l'établissement des règles communes sans pour autant condamner les hommes, mais en s'efforçant de les comprendre. Nous devons nous efforcer « de ne pas rire des actions des hommes, de ne pas les déplorer, 
encore moins de les maudire - mais seulement de les comprendre » (Spinoza, 2005 : 91).

Autrement dit, il nous faut, pour ce qui concerne notre question, établir un ensemble de règles définissant le cadre dans lequel doivent être prises les décisions, non pas en considérant qu'elles seront toujours et nécessairement prises par des hommes uniquement guidés par la raison, mais par des individus mus par des affects sur lesquels ils n'ont pas nécessairement d'emprise. La question de la décision s'apparente donc à la question politique, qui consiste à instaurer des institutions telles qu'elles permettent à des hommes qui ne sont pas naturellement vertueux de vivre comme s'ils l'étaient foncièrement :

Un État, par conséquent, dont le salut dépend de la loyauté de tel ou tel, et dont les affaires ne peuvent être correctement prises en charge qu'à la condition que ceux qui les traitent aient la volonté d'agir loyalement, n’aura aucune stabilité. Pour qu'il puisse durer, au contraire, les affaires publiques doivent être ordonnées de telle sorte que ceux qui les administrent, qu'ils soient conduits par la raison ou par les affects, ne puissent être à manquer de loyauté, ou à mal agir (Spinoza, 2005 : 93).

Pour ce qui concerne la décision médicale, il s'agit, dans une certaine mesure, de définir une procédure délibérative et décisionnelle qui aiderait des hommes qui ne sont pas toujours guidés par la raison à faire les choix les plus rationnels possible. Il serait en effet totalement irréaliste de vouloir que tous les intervenants agissent de manière totalement rationnelle et adoptent tous une attitude raisonnable. $\mathrm{Si}$ « la raison peut sans doute beaucoup pour contenir et régler les affects : mais nous avons vu en même temps que la voie qu'enseigne la raison elle-même est très difficile » (Spinoza, 2005: 93) et ne peut être parcourue que par une infime minorité. Or, fort heureusement, on ne demande pas à un soignant ou à un médecin d'avoir atteint la sagesse suprême et la béatitude pour exercer son art. Il faut donc trouver d'autres voies pour parvenir, malgré tout, à des décisions qui vont dans le sens de l’intérêt des malades et de leurs proches.

\section{Le voile d'ignorance}

Il est donc permis de s'interroger sur la possibilité de recourir à une démarche procédurale comparable à celle que préconise John Rawls dans sa Théorie de la justice (1987). Il peut sembler paradoxal de recourir à une telle méthode pour traiter la question de la délibération à partir d'un postulat déterministe. Mais comme nous l'avons précédemment souligné, dans l'acte même de décider, nous sommes comme contraints de nous situer dans le cadre d'une fiction, qui peut d'ailleurs être consciente d'elle-même, qui nous permet de délibérer et d'agir comme si nous usions d'une volonté disposant d'une relative liberté. Il est certes vrai que, plus notre conscience des déterminations qui nous meuvent est grande, moins cette contrainte s'avère nécessaire, mais comme nous ne pouvons jamais totalement connaître les causes dont nous sommes les objets, il faut toujours y recourir un tant soit peu.

En conséquence, une hypothèse comme celle du voile d'ignorance peut, dans une certaine mesure, assez bien fonctionner, même dans un tel contexte. Cette procédure consiste à délibérer dans le cadre d'une situation hypothétique où chacun ne peut savoir s'il sera ou non favorisé par la décision prise. Il s'agit donc d'aboutir à une décision qui bénéficie à tous, même aux plus mal lotis. Cette procédure pourrait, comme le souligne Suzanne Rameix (1996: 73-76), s'appliquer dans une décision relative à l'attribution d'un organe pour une transplantation lorsqu'il y a plusieurs 
receveurs potentiels. La décision pourra être jugée légitime, même par ceux qui n’ont pas bénéficié de la transplantation, si ces derniers considèrent qu'ils auraient pu effectuer un choix identique en se situant sous le voile d'ignorance.

On pourrait certes rétorquer que cette solution n'a de valeur que théorique et ne peut s'appliquer que dans des situations abstraites et hypothétiques et que, dans la réalité, le médecin ou le soignant n'agit pas dans le cadre d'une expérience de pensée, mais dans des conditions concrètes où il se trouve le plus souvent pressé par l'urgence. Les équipes soignantes n'ont donc guère le temps de se situer intellectuellement dans de telles conditions en envisageant ce type de médiation procédurale : elles doivent plutôt affronter le réel dans son immédiateté et sa brutalité la plus manifeste.

Mais c'est peut-être ici que le caractère rétrospectif de la réflexion joue son rôle, car à force de réexaminer les décisions qu'il a pu prendre ou auxquelles il a pu participer, le sujet est conduit à renouveler sans cesse ce que l'on pourrait appeler sa base de données internes afin d'éclairer chacune de ces décisions par les précédentes. Il est ainsi conduit à prendre en considération non seulement les déterminations externes (contextes social, personnel et professionnel qui l'ont conduit à prendre une décision), mais aussi ses déterminations internes, c'est-à-dire les acquis de son expérience existentielle de la décision.

Il semble donc important, dans les procédures à mettre en place pour faciliter la décision, de prévoir non seulement un dialogue préalable à la décision, mais également un temps pour que les équipes puissent analyser a posteriori les décisions qui ont été prises. Non pour procéder à un examen de conscience, mais pour comprendre les conditions dans lesquelles ont été conduites les délibérations et ont été prises les décisions pour en évaluer les conséquences en fonction des points de vue des différents acteurs et aussi pour tenter de définir les éléments qui ont pu déterminer telle ou telle décision et contribuer à son succès ou au contraire entraîner des conséquences dont les bienfaits peuvent donner lieu à un questionnement. Nous rejoignons ici ce qu'écrit à ce sujet Pierre Le Coz :

Il y a un moment où les émotions sont moins vives et plus accessibles à la
révision : une fois que la décision est devenue irréversible : une fois que « les
dés ont été jetés ». Quand la décision a été prise et qu'il n'est plus possible de
faire marche arrière, alors on peut revenir sur le processus qui y a conduit et le
décomposer pour mieux le comprendre. Le « moment philosophique de la
décision médicale » désigne précisément ce moment de réflexion rétrospective
sur le processus de maturation décisionnelle auquel les acteurs de soin ont
participé. La prise de décision, une fois reprise de façon distanciée, constitue
une expérience fructueuse capable de rejaillir avantageusement sur les
décisions ultérieures (2007:20).

C'est aussi dans ce cadre rétrospectif que pourront être abordées les questions concernant la répartition des responsabilités et la manière dont ont pu être explicitement ou implicitement désignés ceux à qui la décision peut être directement imputée, ainsi que les valeurs à partir desquelles la décision a été prise. C'est en effet une illusion de croire que les valeurs déterminent la décision : il est plus réaliste d'admettre que c'est la décision qui fixe les valeurs. Or, ce qu'il faut éviter, c'est de décider en fonction de ce qui convient le mieux à l'équipe soignante ou à certains de ses membres, plutôt qu'en fonction de ce qui convient au malade et à son entourage, et surtout de croire faire le bien du patient alors qu'on ne vise que ce que l'on croit être le sien propre. Car il n'y a pas tant de distance que cela entre ce qui est bon pour le malade et ce qui est bon pour le soignant. Le soignant ne s'accomplit vraiment que par sa capacité à décrypter ce que désire réellement le malade. Le médecin ou le soignant, s'ils sont autre chose que des « ingénieurs du corps », pour reprendre l'expression de 
Rony Brauman (cité par Todorov, 2007), n'ont d'autre souci que le bien du patient. Cependant, si tous les hommes recherchent ce qui leur est vraiment utile, et si rien n'est plus utile à un homme qu'un autre homme, chacun n'y voit pas toujours clair quant au chemin à suivre pour atteindre ce qui est utile, tant pour soi-même que pour autrui. Par exemple, là où le médecin considérera qu'il n'y a d'autre chemin pour le malade que la guérison, même si celle-ci exige souvent de sa part le renoncement à ce qui donne sens à sa vie, ce dernier pourra préférer voir sa maladie s'installer dans une certaine chronicité, au risque de voir diminuer son espérance de vie, sans avoir à subir des traitements l'empêchant de faire ce sans quoi sa vie ne vaudrait plus pour lui la peine d'être vécue.

Si la connaissance scientifique est toujours science du général, la connaissance du patient est science du singulier. Il faut donc s'efforcer de comprendre ce qui le détermine, les affects qu'il ressent et les idées qui leur sont corrélées pour tenter de décider ce qui convient le mieux à tel patient plutôt qu'à tel autre. Mais ce qu'il faut également examiner, ce sont les affects qui ont pu déterminer les choix des membres de l'équipe soignante. Il convient cependant d'éviter tout contresens et de ne pas transformer ce qui pourrait conduire à une démarche féconde d'aide à la décision en un tribunal dans lequel chacun devrait procéder à son examen de conscience.

\section{Les principes d'une telle conception de la délibération médicale}


délibération et de la décision qu'elle engendre pour mieux viser l'utile propre du patient, c'est-à-dire ce qui pourra l'aider à mieux affirmer ce qui lui reste de puissance d'être et d'agir. $\mathrm{Si}$ « rien n'est plus utile à un homme qu'un autre homme »3 , délibérer et prendre une décision dans le domaine médical consiste à s'efforcer de se rendre utile à cet autre qu'est le patient en essayant de déterminer avec lui et ses proches ce qui lui convient le mieux. C'est pourquoi, même s'il était tentant de considérer que c'est en dernière instance au patient, ou aux personnes de confiance, de décider, il convient de nuancer une telle position.

Le patient n'ayant pas toujours une perception claire de ses propres désirs, il s'agit, lorsque cela est possible, de l'aider à mieux se comprendre lui-même au cours du processus de délibération. Par exemple, le patient qui refuse un soin ou un traitement peut très bien changer d'avis au cours des différentes étapes qui initialement devaient conduire à la mise en place de soins palliatifs et finalement décider de reprendre le protocole qu'il avait d'abord choisi d'abandonner. Cette procédure décisionnelle participe donc à la conquête par le patient d'une certaine autonomie, d'une capacité d'autodétermination qui ne relève cependant pas d'un utilitarisme individualiste qui perçoit le sujet comme un atome séparé de son environnement culturel et social et qui ne tient absolument pas compte du fait qu'il est aussi une personne vulnérable. Il s'agit au contraire de l'élaboration progressive d'une autonomie reposant sur la constitution d'un lien de solidarité unissant le patient et ses proches à l'équipe soignante.

\section{Conclusion}

Ainsi reprise dans le cadre d'une démarche réflexive, la décision reprise régulièrement dans le cadre d'une démarche délibérative ne donne cependant pas lieu à une attitude irrésolue. Toujours orientée en vue de l'utile propre du patient, elle se précise au fur et à mesure qu'elle se constitue. Elle permet au contraire d'éviter que la résolution, qui est nécessaire au passage de la décision à l'action, se dégrade et dégénère en obstination déraisonnable.

Une telle démarche, si elle peut sembler initialement descriptive, n'en est pas moins normative, dans la mesure où elle dessine, dans le cadre de la constitution d'une autonomie solidaire ${ }^{4}$ du patient ou de ses proches, un horizon duquel émergera progressivement ce qui relève pour tous du préférable.

\section{Bibliographie}

ARISTOTE (1990), Éthique à Nicomaque, traduction de J. Tricot, Paris, Vrin.

Delassus, Éric (2011), De l'éthique de Spinoza à l'éthique médicale, Rennes, Presses universitaires de Rennes.

HоввеS, Thomas (2000), Léviathan, traduction, introduction, notes et notices de Gérard Mairet, Paris, Gallimard. (Coll. « Folio essais ».)

LAGRÉE, Jacqueline (2002), Le médecin, le malade et le philosophe, Paris, Bayard.

Le Coz, Pierre (2007), Petit traité de la décision médicale, Paris, Seuil.

Mouiluie, Jean Marc, Céline LefÈve et Laurent Visier (dir) (2007), Médecine et sciences humaines : manuel pour les études médicales, Préface de Tzvetan ToDorov, Paris, Les Belles Lettres.

RameIx, Suzanne (1996), Fondements philosophiques de l'éthique médicale, Paris, Ellipses.

Rawls, John (1987), Théorie de la justice, Paris, Seuil. 
SpINOzA (1988), L'éthique, texte original et traduction nouvelle de Bernard Pautrat, Paris, Seuil.

SPINOZA (1999), Traité de l'amendement de l'intellect, traduction de Bernard Pautrat, Paris, Allia.

SPINOza (2005), Traité politique, texte établi par Omero Proietti, traduction, introduction, notes, glossaires, index et bibliographie de Charles Ramond, Paris, Presses universitaires de France.

\title{
Notes
}

1 Voir « L’homme pense » dans Spinoza (1988: 95).

2 «Car l'intellect et la volonté qui constitueraient l'essence de Dieu devraient différer de notre intellect et de notre volonté de toute l'étendue du ciel, et ne pourraient avoir avec eux, d'autre convenance que de nom : pas autrement qu'il y a convenance entre le chien, signe céleste, et le chien, animal aboyant » (Spinoza, $1988: 49)$.

3 À l'homme donc, rien de plus utile que l'homme : il n'est rien dis-je, que les hommes puissent souhaiter de mieux pour conserver leur être que de se convenir tous en tout, en sorte que les Esprits et les Corps de tous composent pour ainsi dire un seul Esprit et un seul Corps, de s'efforcer de tous ensemble de conserver leur être, autant qu'ils peuvent, et de rechercher tous ensemble et chacun pour soi l'utile qui est commun à tous : d'où il suit que les hommes que gouverne la raison, n'aspirent pour eux-mêmes à rien qu'ils ne désirent pour tous les autres hommes, et par suite sont justes, de bonne foi et honnêtes » (Spinoza, 1988 : 371 ).

4 Voir à ce sujet la conclusion de mon livre De l'éthique de Spinoza à l'éthique médicale, dans laquelle je développe ce concept d'autonomie solidaire (2011 : 319-323).

\section{Pour citer cet article}

Référence électronique

Éric Delassus, « La délibération comme démarche réflexive accompagnant la décision médicale », Éthique publique [En ligne], vol. 16, n 2 | 2014, mis en ligne le 12 janvier 2015, consulté le 12 mai 2015. URL : http://ethiquepublique.revues.org/1569 ; DOI :

10.4000/ethiquepublique. 1569

\begin{abstract}
Auteur
Éric Delassus

Professeur agrégé et docteur en philosophie, Éric Delassus enseigne au lycée Marguerite de Navarre de Bourges. Il est membre du Laboratoire de recherche en éthique médicale de la Faculté de médecine de Tours et du Groupe d'aide éthique du centre hospitalier de Bourges. Ses recherches portent principalement sur les questions d'éthique contemporaine : éthique médicale, éthique et relations humaines dans le monde du travail, éthique et esthétique. Spécialiste de Spinoza, il travaille principalement sur les usages contemporains de sa pensée en lien avec l'éthique du care. Sa thèse de doctorat, De l'éthique de Spinoza à l'éthique médicale, a été publiée en 2011 aux Presses universitaires de Rennes. Éric Delassus a également publié en septembre 2014 un recueil de ses articles en deux volumes aux Éditions L'Harmattan intitulé Sagesse de l'homme vulnérable ainsi qu'un commentaire de l'appendice à la première partie de l'Éthique de Spinoza aux Éditions Boréal en novembre 2014.
\end{abstract}

\section{Droits d'auteur}

Tous droits réservés 